J. Plasma Physics (2018), vol. 00, part 0,pp. 1-000. (C) 2018 Cambridge University Press

DOI: $10.1017 /$ S0000000000000000 Printed in the United Kingdom

\title{
Compressive high-frequency waves riding on an Alfvén-cyclotron wave in a multi-fluid plasma
}

\author{
DANIEL VERSCHAREN † and ECKART MARSCH \\ Max-Planck-Institut für Sonnensystemforschung, Max-Planck-Straße 2, \\ D-37191 Katlenburg-Lindau, Germany
}

(Received 3 December 2010, revised 3 February 2011, accepted 4 February 2011)

\begin{abstract}
We study weakly-compressive high-frequency plasma waves which are superposed on a large-amplitude Alfvén wave in a multi-fluid plasma consisting of protons, electrons, and alpha particles. For these waves, the plasma environment is inhomogeneous due to the presence of the low-frequency Alfvén wave with large amplitude, a situation that may apply to space plasmas such as the solar corona and solar wind. The dispersion relation of the plasma waves is determined from a linear stability analysis using a new eigenvalue method that is employed to solve the set of differential wave equations which describe the propagation of plasma waves along the direction of the constant component of the Alfvén wave magnetic field. This approach allows one to consider also weak compressive effects. In the presence of the background Alfvén wave, the dispersion branches obtained differ significantly from the situation of a uniform plasma. Due to compressibility, acoustic waves are excited and couplings between various modes occur, and even an instability of the compressive mode. In a kinetic treatment, these plasma waves would be natural candidates for Landau-resonant wave-particle interactions, and may thus via their damping lead to particle heating.
\end{abstract}

\section{Introduction}

The solar wind is the classical paradigm of a nonuniform plasma which is structured in space and time (Schwenn and Marsch, 1991) and permeated by fluctuations on a wide range of scales. It reveals turbulence and carries large-amplitude Alfvén waves which mostly originate in the solar corona (for reviews of the magnetohydrodynamic turbulence see, e.g., Tu and Marsch (1995); Bruno and Carbone (2005) and the plasma kinetics see Marsch (2006)). The plasma waves in the solar wind are thus riding on a varying background, and throughout their passage from the corona the solar wind particles are observed to evolve non-adiabatically, and therefore are supposed to undergo continuous heating out to 1 AU (e.g. Kasper et al., 2008) and beyond. It is believed since a long time (Coleman, 1968; Belcher and Davis, 1971) that wave-particle interactions (Marsch, 2006) with the ubiquitous fluctuations in the electromagnetic fields are the main driver of this heating process. Here we study

$\dagger$ verscharen@mps.mpg.de

$\ddagger$ marsch@mps.mpg.de 
weakly-compressive high-frequency plasma waves which are superposed on a largeamplitude Alfvén wave in a multi-fluid plasma consisting of protons, electrons, and alpha particles like in the solar wind. The term 'high-frequency wave' refers to the wavenumber regime around the inverse ion inertial length $\ell_{\mathrm{p}}$ in contrast to the non-dispersive low-frequency MHD limit.

As is well known, there are two important kinetic resonances that can lead to dissipative heating of a plasma by wave-particle interactions with waves that propagate parallel to the background magnetic field. The first is Landau resonance requiring a parallel wave electric field, and the second cyclotron resonance (e.g. Akhiezer et al., 1975; Hollweg and Isenberg, 2002) that couples to the perpendicular wave electric field. The condition for Landau resonance is given by $k v_{\|}-\omega=0$, where $k$ denotes the parallel wave number, $v_{\|}$the particle velocity in the direction parallel to the background magnetic field and $\omega$ the wave frequency. This effect can lead to parallel heating of the particles (Lehe et al., 2009). In a low-beta plasma such as the solar corona, it is difficult to fulfill this condition if the wave phase speed is close to the Alfvén speed, because $v_{\text {th }} \ll V_{\mathrm{A}}$ (Chandran et al., 2010).

The cyclotron resonance is connected with the transverse electromagnetic field, and the resonance condition is given by $k v_{\|}-\omega-n \Omega_{j}=0$ with an integer $n$ and the particle gyrofrequency $\Omega_{j}$ (only $n=1$ is allowed in the case of parallel propagation). This resonance can lead to pitch-angle diffusion which is indeed observed in solar wind protons (Heuer and Marsch, 2007). The perpendicular fluctuations must have high wave numbers in the range of the gyroradius to fulfil the resonance condition. Alfvén/ion-cyclotron waves (A/ICs) are possible candidates for waves that can undergo this kind of interaction. However, their origin and evolution are not fully understood, even though their existence in the solar wind was recently proven (Jian et al., 2009). Indirect evidence for Alfvén-cyclotron heating was already referred to by several authors beforehand from simulations (Gary et al., 2005) and proton in-situ observations (Marsch and Tu, 2001; Kasper et al., 2008).

The role of weakly compressive waves in the different phenomena of plasma heating is currently under wide discussion (Tu and Marsch, 1994; Chandran, 2005; Bale et al., 2005; Kellogg et al., 2006; Chandran et al., 2009; Verdini et al., 2010). Especially, kinetic Alfvén waves (KAWs) have come into the focus of the debate, because they are both transverse and compressive. However, several problems also arise from this interpretation of solar wind fluctuations, especially at high wave numbers (Podesta et al., 2010).

It has also been known for a long time that compressibility plays a major role in the context of the parametric instabilities of large-amplitude waves (Galeev and Oraevskii, 1963; Goldstein, 1978; Lashmore-Davies and Stenflo, 1979; Stenflo and Shukla, 2007). These instabilities are always connected with compressive components of the daughter-wave products. Our subsequent analysis is based on the previous derivations by Marsch and Verscharen (2011), which treat the density fluctuations in terms of the longitudinal electrostatic field and a ponderomotive electric field. The connection between density fluctuations and ponderomotive forces in the context of parametric instabilities was discussed before by Sharma and Shukla (1983) for many different wave modes. However, the work of those authors was focused on frequencies around the upper-hybrid frequency, which is much higher than the frequencies considered here, and thus beyond the scope of this paper. Electromagnetic circularly polarised waves can also be generated from the high-frequency side. For example, Murtaza and Shukla (1984) discussed how an upper-hybrid wave 
can generate such waves in a two-fluid model. For this purpose, electrostatic effects and ponderomotive forces had to be included in the model describing the high-frequency pump wave. The importance of electrons in the decay of compressional Alfvén waves was discussed more recently by Brodin et al. (2008) in terms of the Hall-MHD description. These authors found a new decay channel for oblique daughter waves, and discovered that the wave decay products could grow on scales around the ion inertial length. They also discussed the role of kinetic Alfvén waves as decay products and their possible ability to heat the plasma.

Our present work concentrates on purely parallel wave propagation, but we consider the role of both electrostatic and electromagnetic components of the wave modes in a multi-fluid plasma. The nonlinear coupling of the longitudinal electrostatic field and of the ponderomotive electric fields with the transversal electromagnetic wave fields is the main reason for the significant changes we found in the mode structure and polarisation. However, the modified wave modes and the possible decay products are, due to the given geometry, still forced to propagate along the mean field which is determined by the constant longitudinal magnetic-field component. So we do not consider genuine oblique wave propagation.

The multi-fluid wave equations are solved here with an eigenvalue and eigenvector method that opens a new and unusual way of analysing the pump wave decay and dispersion properties of the resulting plasma waves. This approach also provides the dispersion and polarisation properties of these waves in a comprehensible and direct way.

As mentioned above, it has been known for a long time that the solar wind is permeated by waves and structures on many different scales (Tu and Marsch, 1995). But also in the solar corona low-frequency waves in the magnetic field were recently observed by remote-sensing techniques (De Pontieu et al., 2007). Therefore, in theory and modelling it is obviously necessary to assume an inhomogeneous background magnetic field. The natural choice for such a field might be one consisting of low-frequency Alfvén waves.

Consequently, the scenario assumed for our present theoretical treatment is the following. A low-frequency Alfvén-cyclotron wave is assumed to provide the nonuniform background magnetic field and corresponding background velocity field, according to the wave polarisation relation. In the flank of this wave, a linear dispersion and stability analysis is performed for a three-component plasma consisting of protons, electrons, and alpha particles, whereby also drifts among these species with respect to each other can be included. The situation is sketched in Fig. 1. The applied multi-fluid model allows to consider transverse waves with an intrinsic weakly compressive component. If transverse dispersion branches at wave numbers close to the ion gyroradius are found, then these can be made responsible for possible perpendicular ion heating. Their compressive electrostatic components can in turn explain parallel heating. Since the observations indicate large perpendicular temperature anisotropies in the corona (Antonucci et al., 2000; Kohl et al., 2006) and in fast solar wind (Marsch, 2006), the assumption of weak compressibility seems to be justified empirically, and is also consistent with the measured density fluctuation level (Tu and Marsch, 1995). 


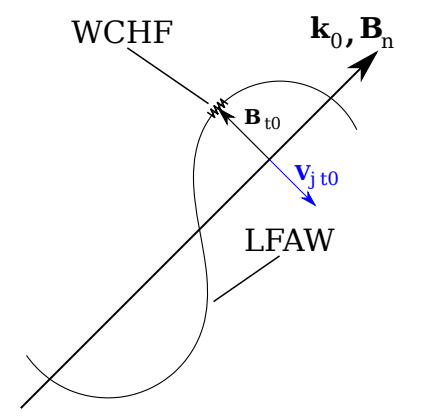

Figure 1. Geometry of the scenario. On a constant magnetic field $\boldsymbol{B}_{\mathrm{n}}$ along the axis of propagation $\boldsymbol{k}_{0}$, a low-frequency Alfvén wave (LFAW) propagates and generates the transverse background fields $\boldsymbol{B}_{\mathrm{t} 0}$ and $\boldsymbol{V}_{j \mathrm{t} 0}=-\zeta \boldsymbol{B}_{\mathrm{t} 0}$ with $\zeta=$ const. $>0$. Weakly-compressive high-frequency waves (WCHF) are superposed in the flank of the Alfvén wave.

\section{Theoretical approach and numerical treatment}

\subsection{The multi-fluid model}

Following the detailed derivations of Marsch and Verscharen (2011), we obtain subsequently a set of second-order ordinary coupled differential equations (DEs) of the relevant fields for parallel propagation, in which case $B_{\mathrm{n}}$ is a conserved quantity, and thus the fields can be made dimensionless as follows: $\boldsymbol{v}_{j \mathrm{t}}=\boldsymbol{V}_{j \mathrm{t}} / V_{\mathrm{A}}$, and $\boldsymbol{b}_{\mathrm{t}}=\boldsymbol{B}_{\mathrm{t}} / B_{\mathrm{n}}$. Similarly, we define the normalised longitudinal electric fields as follows:

$$
e_{j \mathrm{n}}=\frac{c E_{j \mathrm{n}}}{B_{\mathrm{n}} V_{\mathrm{A}}}, \quad e_{\mathrm{n}}=\frac{c E_{\mathrm{n}}}{B_{\mathrm{n}} V_{\mathrm{A}}} .
$$

Length scales can be all normalised in units of the ion inertial length scale $\ell_{\mathrm{p}}=$ $c / \omega_{\mathrm{p}}$. In the following, all velocities are normalised to the proton Alfvén speed $V_{\mathrm{A}}=B_{\mathrm{n}} / \sqrt{4 \pi n_{\mathrm{p}} m_{\mathrm{p}}}$ and then denoted by lower case letters. This choice is different than the normalisation by Marsch and Verscharen (2011) but it is more appropriate here, because the wave phase speed $V$ will be used as a free parameter for different evaluations of the wave equations. Therefore, it is better to normalise with a fixed velocity. The natural choice for such a velocity is the proton Alfvén speed. In this normalisation system, frequencies are normalised to the proton gyration frequency $\Omega_{\mathrm{p}}$, which is equal to $V_{\mathrm{A}} / \ell_{\mathrm{p}}$. In the subsequent paper, we use $\Omega_{j}$ as the normalised gyration frequency of species $j$. We also recall the original definition

$$
e_{j \mathrm{n}}=\frac{1}{c}\left(\boldsymbol{v}_{j \mathrm{t}} \times \boldsymbol{b}_{\mathrm{t}}\right) \cdot \hat{\boldsymbol{n}} .
$$

Using the same normalisation for the transverse electric field, we obtain

$$
\boldsymbol{e}_{\mathrm{t}}=-v\left(\hat{\boldsymbol{n}} \times \boldsymbol{b}_{\mathrm{t}}\right),
$$

which is fully determined by the solution found for $\boldsymbol{b}_{\mathrm{t}}$, which means it is a dependent auxiliary field. Completing the required definitions, we quote the differential operator

$$
\mathcal{D}_{j}=\frac{\mathrm{d}^{2}}{\mathrm{~d} \xi^{2}}+\frac{1}{r_{j}^{2}},
$$

where we have defined the spatial coordinate along $B_{\mathrm{n}}$ as $\xi_{\mathrm{n}}=\xi$, which we will continue using subsequently. After these preparations we can state the DEs, as derived from the equations for parallel wave propagation. Firstly, we obtain for each 
particle species with respect to its transverse motion a forced harmonic oscillator equation reading

$$
\mathcal{D}_{j} \boldsymbol{v}_{j \mathrm{t}}=\left(\frac{e_{\mathrm{n}}+e_{j \mathrm{n}}}{c_{j}^{2}-v_{j \mathrm{n}}^{2}} \frac{\mathrm{d} \boldsymbol{v}_{j \mathrm{t}}}{\mathrm{d} \xi}+\frac{\boldsymbol{b}_{\mathrm{t}}}{r_{j}}+\hat{\boldsymbol{n}} \times \frac{\mathrm{d} \boldsymbol{b}_{\mathrm{t}}}{\mathrm{d} \xi}\right) \Omega_{j} .
$$

Secondly, one can rewrite the mutually coupled and driven wave equations for the transverse magnetic field,

$$
\mathcal{D}_{B} \boldsymbol{b}_{\mathrm{t}}=-\sum_{j} \frac{1}{\ell_{j}^{2}}\left(\frac{\boldsymbol{v}_{j \mathrm{t}}}{v_{j \mathrm{n}}}+\frac{\left(e_{\mathrm{n}}+e_{j \mathrm{n}}\right)}{c_{j}^{2}-v_{j \mathrm{n}}^{2}}\left(\hat{\boldsymbol{n}} \times \boldsymbol{v}_{j \mathrm{t}}\right)\right),
$$

and the longitudinal electric field,

$$
\mathcal{D}_{E} e_{\mathrm{n}}=\sum_{j} \frac{e_{j \mathrm{n}}}{\lambda_{j}^{2}}
$$

All involved parameters such as $v_{j \mathrm{n}}, c_{j}, \lambda_{j}, \ell_{j}$ and $r_{j}$ have non-vanishing mean values, as the density $n_{j}$ is always nonzero and its fluctuations throughout are assumed to be small. Note that $\Omega_{j}$ is strictly constant as it depends on the conserved quantity $B_{\mathrm{n}}$. The other parameters can be calculated by use of the background number density $\bar{n}_{j}$, as well as by exploiting the conditions for quasi-neutrality, zero longitudinal current, and zero centre-of-momentum velocity (Marsch and Verscharen, 2011). Compression is accounted for solely by the longitudinal electric field in this approximation. This is the major advantage of this multi-fluid system.

Furthermore, the multi-fluid approach incorporates the natural scales of the plasma and allows therefore the treatment of dispersive waves in the high-frequency range which is not accessible by MHD considerations. The compressibility can be accounted for in a comparably lucid way through the electric field variables $e_{\mathrm{n}}$ and $e_{j \mathrm{n}}$. The nonlinear equations (2.5), (2.6), and (2.7) form a closed set of DEs, which describe the leading-order compressive effects via $e_{\mathrm{n}}(\xi)$. Before we write down these field equations in their components, let us define an appropriate coordinate system. For a right-handed orthogonal system, we choose the unit vectors $\hat{\boldsymbol{n}}=\boldsymbol{e}_{3}=\boldsymbol{e}_{1} \times \boldsymbol{e}_{2}$, $\boldsymbol{e}_{1}=\boldsymbol{e}_{2} \times \hat{\boldsymbol{n}}$, and $\boldsymbol{e}_{2}=\hat{\boldsymbol{n}} \times \boldsymbol{e}_{1}$. The normalised (dimensionless) transverse magnetic field is

$$
\boldsymbol{b}_{\mathrm{t}}=b_{1}(\xi) \boldsymbol{e}_{1}+b_{2}(\xi) \boldsymbol{e}_{2} \text {. }
$$

Similarly, the transverse flow velocity of any species $j$ is given by

$$
\boldsymbol{v}_{j \mathrm{t}}=v_{j 1}(\xi) \boldsymbol{e}_{1}+v_{j 2}(\xi) \boldsymbol{e}_{2} .
$$

The particle speed along the direction of propagation in the fixed coordinate system (not in the co-moving frame) is denoted by $u_{j n}$. If there are no differential drift motions along the mean magnetic field in the background plasma, i.e., if for all $j$ we have $u_{j \mathrm{n}}=0$, then $v_{j \mathrm{n}}=-v$, and thus the longitudinal gyration length becomes $r_{j}=-v / \Omega_{j}$, which by definition is not a positive-definite quantity as the gyrofrequency carries the sign of the charge of the species considered. Using Eq. (2.8) and Eq. (2.9), the longitudinal electric field associated with species $j$ simply reads

$$
e_{j \mathrm{n}}=v_{j 1} b_{2}-v_{j 2} b_{1} .
$$

We recall from Marsch and Verscharen (2011) that $e_{j n}$ can also be expressed as the gradient of a potential, which is given by the transverse kinetic energy of species $j$ 
and then has the normalised form,

$$
e_{j \mathrm{n}}=-\frac{1}{\Omega_{j}} \frac{\mathrm{d}}{\mathrm{d} \xi}\left(\frac{1}{2} \boldsymbol{v}_{j \mathrm{t}}^{2}\right) .
$$

In terms of components, we have from a comparison of that equation with Eq. (2.10) the relation,

$$
-\frac{1}{\Omega_{j}} \frac{\mathrm{d} v_{j 1,2}}{\mathrm{~d} \xi}= \pm b_{2,1},
$$

which we can use to replace the first derivative of the transverse velocity by the magnetic field. Written out explicitly, the set of DEs in dimensionless form finally reads as follows:

$$
\begin{aligned}
\frac{\mathrm{d}^{2} v_{j 1,2}}{\mathrm{~d} \xi^{2}}+\frac{v_{j 1,2}}{r_{j}^{2}} & =\left[\frac{b_{1,2}}{r_{j}} \mp \frac{\mathrm{d} b_{2,1}}{\mathrm{~d} \xi} \mp \frac{e_{n}+e_{j \mathrm{n}}}{c_{j}^{2}-v_{j \mathrm{n}}^{2}} b_{2,1}\right] \Omega_{j} \\
\frac{\mathrm{d}^{2} b_{1,2}}{\mathrm{~d} \xi^{2}}-\frac{b_{1,2}}{\ell_{\mathrm{S}}^{2}} & =-\sum_{j} \frac{1}{\ell_{j}^{2}}\left[\frac{v_{j 1,2}}{v_{j \mathrm{n}}} \mp \frac{e_{n}+e_{j \mathrm{n}}}{c_{j}^{2}-v_{j \mathrm{n}}^{2}} v_{j 2,1}\right] \\
\frac{\mathrm{d}^{2} e_{\mathrm{n}}}{\mathrm{d} \xi^{2}}-\frac{e_{\mathrm{n}}}{\lambda_{\mathrm{D}}^{2}} & =\sum_{j} \frac{1}{\lambda_{j}^{2}}\left(v_{j 1} b_{2}-v_{j 2} b_{1}\right)
\end{aligned}
$$

The operators on the left-hand sides of Eqs. (2.13), (2.14), and (2.15) describe the dynamics of the uncoupled free fields and have a simple physical interpretation. After Fourier transformation (yielding $\mathrm{d} / \mathrm{d} \xi \rightarrow i k$ ) the solution of Eq. (2.13) gives a helical gyration in space of the transverse velocity about the mean field $B_{\mathrm{n}}$ with wave vector $k= \pm 1 / r_{j}$, the solution of Eq. (2.14) corresponds to diamagnetism, i.e. the static penetration of the transverse field into the plasma by the skin depth $\ell_{\mathrm{S}}=c / \omega_{\mathrm{P}}$, and Eq. (2.15) gives for $v=0$ the static screening by the Debye length $\lambda_{\mathrm{D}}$, or for finite speed $v$ the electrostatic wave dispersion relation, $\left(k \lambda_{\mathrm{D}}\right)^{2}+1=0$. This, for zero drifts, transforms into the electrostatic dispersion relation

$$
k^{2}=\sum_{j} \frac{\omega_{j}^{2}}{v^{2}-c_{j}^{2}},
$$

the zeroes of which which yield the Langmuir and ion-acoustic waves. For finite right-hand sides of the above DEs, the fields are coupled (note that the abbreviation from Eq. (2.10) has to be included). The incompressible limit (with $e_{\mathrm{n}}=e_{j \mathrm{n}}=0$ ) then gives the monochromatic (only a single $k=k(V)$ is permitted from the dispersion relation) electromagnetic Alfvén-cyclotron wave that has a constant but arbitrarily large amplitude. When considering compressibility, the electric and electromagnetic waves are linked and interact through the nonlinear rightmost terms in the above wave equations. Considering compressibility may require either a perturbative approach or numerical treatment.

\subsection{Perturbative approach and linearisation}

The above equations are a system of second-order wave equations. To simplify it, we reduce it to a system of first-order equations. Therefore, we introduce the quantities $e_{\mathrm{n}}^{\prime} \equiv \mathrm{d} e_{\mathrm{n}} / \mathrm{d} \xi, b_{1,2}^{\prime} \equiv \mathrm{d} b_{1,2} / \mathrm{d} \xi$, and $v_{j 1,2}^{\prime} \equiv \mathrm{d} v_{j 1,2} / \mathrm{d} \xi$. With this substitution, the system corresponds to a set of 18 coupled nonlinear first-order ordinary differential 
equations in $\xi$ for an electron-proton plasma. For each additional particle species, four coupled equations are added.

In the next step, the system (2.13-2.15) is linearised around a background given by the wave amplitude vectors $\boldsymbol{v}_{j \mathrm{t} 0}$ and $\boldsymbol{b}_{j \mathrm{t} 0}$ to determine the wave dispersion. A suitable choice of the background values is given in Sect. 2.3.

Nonlinear couplings of low-frequency waves with fluctuations at high frequencies cannot be described by a linearised system. The low-frequency wave with wave number $k_{0}$, however, may be treated as a constant inhomogeneous background if the high-frequency waves with wave numbers $k$ fulfill the condition $k_{0} \ll k$. The high-frequency waves are then treated in the flank of this low-frequency wave that does not change its fields significantly over several periods of the high-frequency waves. The particle velocities, the electromagnetic field, and their derivatives are combined in a state vector,

$$
\boldsymbol{y}=\left(v_{\mathrm{p} 1}^{\prime}, v_{\mathrm{p} 2}^{\prime}, \ldots, v_{\mathrm{p} 1}, v_{\mathrm{p} 2}, \ldots, b_{1}^{\prime}, b_{2}^{\prime}, b_{1}, b_{2}, e_{\mathrm{n}}^{\prime}, e_{\mathrm{n}}\right),
$$

and therewith one can write the linearised equation as,

$$
\frac{\mathrm{d}}{\mathrm{d} \xi} \delta \boldsymbol{y}=\mathcal{A} \delta \boldsymbol{y},
$$

with the quadratic matrix $\mathcal{A}=\left(a_{i, j}\right) \in M(n)$ with $n=4 s+6$ ( $s$ is the total number of species). The solution of Eq. (2.18) is in general given by

$$
\delta \boldsymbol{y}=\sum_{i=1}^{4 s+6} \alpha_{i} \delta \boldsymbol{y}_{i} e^{\lambda_{i} \xi},
$$

with the eigenvalues $\lambda_{i}$ and the corresponding eigenvectors $\delta \boldsymbol{y}_{i}$. Since $\mathcal{A}$ is a real matrix, the complex conjugated eigenvalues and eigenvectors are also solutions once a complex eigenvalue or eigenvector is found. The coefficients $\alpha_{i}$ are arbitrary. However, they must be equal for the pairwise complex conjugated eigenvalues to construct a real solution.

An imaginary part of an eigenvalue $\lambda_{i}$ always indicates a periodic fluctuation. Due to the rule of pairwise complex conjugated eigenvalues, they are represented by real sine or cosine functions. Real parts of $\lambda_{i}$ correspond to growth or damping. We denote the imaginary part as $k$ and the real part as $\kappa$. If the eigenvalues are pairwise symmetric in the real part, the growth is described by hyperbolic sine or cosine functions, which is the symmetric solution for the instabilities growing in positive and in negative $\xi$-direction.

The eigenvalues can be calculated numerically with the QR-method after transforming $\mathcal{A}$ to an upper Hesse matrix (Press et al., 1992). The existence of the complex-conjugated eigenvalues means that for wave-like daughter products always two solutions exist, one foreward and one backward propagating with the same frequencies.

\subsection{Background wave}

An adequate background to calculate the dispersion is the flank of a circularly polarised Alfvén wave, since these waves are exact eigenmodes of a plasma and obey Eqs. (2.13) and (2.14) in the incompressible limit with arbitrary amplitudes.

The phase speed of this wave is denoted by $v_{0}$. It is left-hand polarised and has a 
magnetic field of the structure

$$
\boldsymbol{b}_{\mathrm{t} 0}=b\left(\begin{array}{c}
\cos \left(k_{0} \xi\right) \\
\sin \left(k_{0} \xi\right)
\end{array}\right)
$$

Without loss of generality, we can evaluate this field at the point $\xi=0$. For sufficient low $k_{0}$-values, this field appears as a constant magnetic background field of the magnitude $\boldsymbol{b}_{\mathrm{t} 0}=(b, 0)$ as stated above. Therefore, this background describes analogous conditions as used to treat oblique propagation of linear modes. However, the wave is additionally associated with a transversal velocity for each species. Corresponding to this magnetic field, a background velocity field occurs that is determined by the polarisation relation of circularly polarised Alfvén waves

$$
\boldsymbol{v}_{j \mathrm{t} 0}=\frac{v_{j \mathrm{n} 0}}{1+k v_{j \mathrm{n} 0} / \Omega_{j}} \boldsymbol{b}_{\mathrm{t} 0}
$$

as it is derived by Sonnerup and $\mathrm{Su}$ (1967) for example. The normal velocity component of the particle species $j$ in the reference frame moving with $v_{0}$ is denoted by $v_{j \mathrm{n} 0}$. The polarisation relation is also in agreement with the results by Marsch and Verscharen (2011). For a sufficient small wavenumber $k_{0}$, the wave fulfills the Alfvénic dispersion relation $v_{0} \simeq v_{\mathrm{A}}$.

The transformation of the transverse velocity to the co-moving reference frame does not change the value for $\boldsymbol{v}_{j \mathrm{t} 0}$. Therefore, the polarisation relation provides the necessary (constant) value for $\boldsymbol{v}_{j \text { to }}$ depending on a given (small) $k_{0}$ and the wave amplitude $b$.

To evaluate the polarisation relation exactly, the dispersion relation for the circularly polarised Alfvén waves is used. It is given by

$$
k_{0}^{2}+\sum_{j=1}^{s} \frac{\omega_{j}^{2}}{c^{2}} \frac{k_{0} v_{j \mathrm{n} 0}}{k_{0} v_{j \mathrm{n} 0}+\Omega_{j}}=0
$$

as the non-relativistic limit from Sonnerup and $\mathrm{Su}(1967)$.

\section{Dispersion of high-frequency waves}

In this section we study the wave dispersion relation of high-frequency waves propagating on the nonuniform background Alfvén wave. The most important free parameter of the system of equations (2.13-2.15) is the disposable wave phase speed $v$. For a fixed $v$, the eigenvalue/eigenvector method provides the corresponding wavenumber values $k(v)$. The relation $\omega=k v$ then delivers the corresponding $\omega$, and thus by scanning through all relevant values of $v$ the full dispersion relation can be determined. The growth rate can be determined similarly by evaluating $\gamma=\kappa v$ with the spatial growth rate $\kappa$ from the eigenvalue determination. The very highfrequency branches, which are dominated by the electron dynamics, are not treated in detail, since the ions carry the main momentum, and their wave-induced motion is therefore more important for the heating and acceleration processes. The electron density and relative velocity with respect to the protons and the other ionic species is determined by the requirement of vanishing charge density (quasineutrality) and 
vanishing constant longitudinal currents of the background, such that

$$
\begin{aligned}
\sum_{j=1}^{s} n_{j} q_{j} & =0, \\
\sum_{j=1}^{s} n_{j} q_{j} v_{j \mathrm{n}} & =0 .
\end{aligned}
$$

The specific-heat ratio is set to its adiabatic value of $\gamma_{j}=5 / 3$ for all species.

\subsection{Homogeneous background}

Before we discuss the case of an inhomogeneous plasma, we apply the system of equations and the linearisation to an electron-proton plasma for a homogeneous background and with $\beta_{j}=0$. The plasma beta is defined as the ratio of the thermal energy density to the magnetic field energy density for each species. The background field is set to $B_{\mathrm{n}}=5 \times 10^{-5} \mathrm{G}$ and the particle number densities to $n_{\mathrm{p}}=n_{\mathrm{e}}=5 \mathrm{~cm}^{-3}$. The ratio $B_{\mathrm{n}}^{2} / n_{\mathrm{p}}$ must be parameterised even in the dimensionless normalisation of the system, in order to fix the normalised plasma frequency $\omega_{j} / \Omega_{\mathrm{p}}$ for all electrostatic processes. The result of the calculation is shown in Fig. 2a. The two incompressible branches correspond to the transverse particle motions. They represent a free motion with the gyration frequency for the protons at $\omega=1$, their normalised gyrofrequency. The branch approaching the gyrofrequency asymptotically corresponds to the Alfvén/ion-cyclotron wave. In Fig. 2, the cold dispersion relation for Alfvén/ion-cyclotron waves

$$
\left(\frac{\omega}{\Omega_{\mathrm{p}}}\right)^{2}=\ell_{\mathrm{p}}^{2} k^{2}+\frac{\ell_{\mathrm{p}}^{4} k^{4}}{2}-\frac{\ell_{\mathrm{p}}^{3} k^{3}}{2} \sqrt{\ell_{\mathrm{p}}^{2} k^{2}+4}
$$

is additionally plotted as green dotted line. The branch at low $k$-values with high phase speed $v$ corresponds to the whistler mode shown as red dashed line. The corrected low-frequency dispersion for the multi-fluid R-mode wave (i.e., the whistler wave with ionic effects) is given analytically by

$$
\omega=\frac{\Omega_{\mathrm{e}}}{2\left(1+\frac{1}{\ell_{\mathrm{e}}^{2} k^{2}}\right)}\left[\sqrt{1+\frac{4}{\ell_{\mathrm{p}}^{2} k^{2}}}+1\right] .
$$

Both relations show a perfect agreement with the numerical calculations made with the eigenvalue method (Stix, 1992). This confirms the validity of our approach.

In Fig. $2 \mathrm{~b}$, the dispersion relation is shown for a plasma consisting of electrons, protons, and alpha particles without any relative drifts and without a background wave field. The particle species have a beta of 0.01 . The proton number density is set to $n_{\mathrm{p}}=5 \mathrm{~cm}^{-3}$, the alpha particle number density to $n_{\alpha}=0.04 n_{\mathrm{p}}$ and the electron number density according to Eq. (3.1). The alpha-particle cyclotron branch approaches asymptotically the frequency $\omega=1 / 2$ as expected. This value corresponds to the alpha particle gyrofrequency in normalised units. The cyclotron branch of the protons is slightly deformed in the low- $k$ range in comparison to the cold plasma dispersion relation. Also the whistler wave mode is slightly shifted. The presence of the alpha particles is responsible for this deviation. Further correction terms would be needed to represent the dispersion relation in this three-fluid plasma analytically.

Our model also allows to include relative drifts of the particles along the wave 

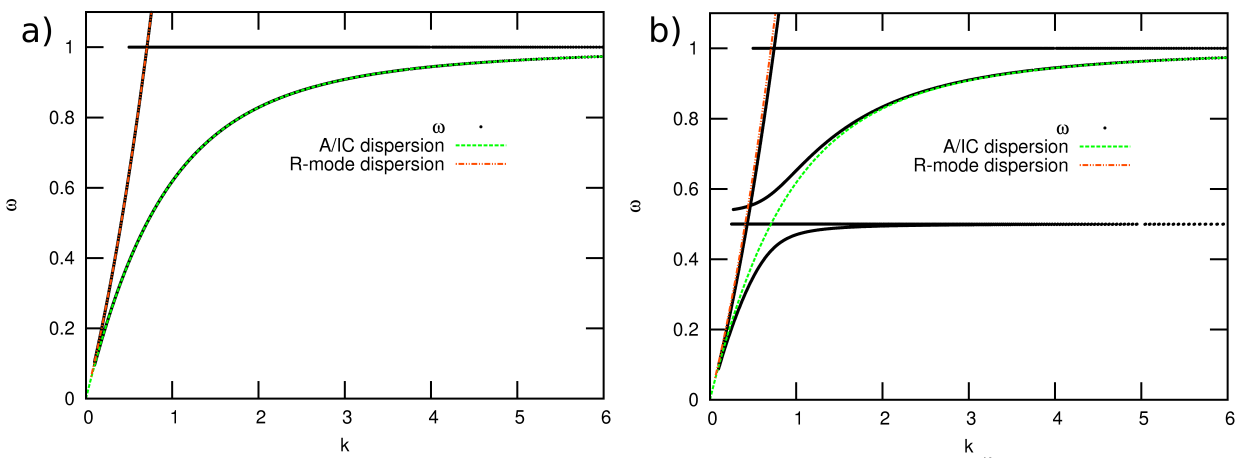

Figure 2. a) Dispersion relation for an electron-proton plasma in a homogeneous background with $\beta=0$. The cold dispersion relation for Alfvén/ion-cyclotron waves and for ion-corrected R-mode waves are additionally plotted. b) The same for a plasma consisting of electrons, protons, and alpha particles with $\beta=0.01$.

normal direction. The relative drift speed between protons and alpha particles is defined as $v_{\mathrm{d}} \equiv v_{\mathrm{pn}}-v_{\alpha \mathrm{n}}$. In Fig. 3 , the dispersion relation for $v_{\mathrm{d}}=0.2$ is shown. The alpha-particle branch starting at $\omega=\Omega_{\alpha}$ is turned into the so-called beam-mode branch (line inclined to the left) derived from the resonance condition

$$
\omega=\Omega_{\alpha}+k u_{\alpha \mathrm{n}}
$$

where $u_{\alpha \mathrm{n}}=v-v_{\alpha \mathrm{n}}$ denotes again the alpha-particle bulk speed component in the wave normal direction in the proton rest frame. For a vanishing drift $u_{\mathrm{n} \alpha}$, this mode is flattened back to the horizontal line $\omega=\Omega_{\alpha}=1 / 2$.

\subsection{Inhomogeneous background}

Next, our system of coupled wave equations can be applied to an inhomogeneous background plasma, which corresponds to realistic solar wind conditions. The plasma consists of three species: protons, electrons, and alpha particles. The relative drift between protons and alphas is set to $v_{\mathrm{d}}=v_{p \mathrm{n}}-v_{\alpha \mathrm{n}}=0.2$, and the plasma beta to $\beta_{j}=0.01$ for each species. The background wave is assumed to have a normalised wave number of $k_{0}=0.01$ and an amplitude of $b=0.1$. Such a wave has a phase speed of almost the local proton Alfvén speed. The wave number is small enough to allow us to neglect any direct nonlinear couplings between the background wave and the high-frequency waves. For the latter, the background wave appears as a quasi-constant field with respect to which the system can be linearised. The results are shown in Fig. 4.

The dispersion branches are deformed for several reasons. The proton Alfvén/ioncyclotron branch at lower wave numbers is not further deformed as compared to Fig. 2b. Some branches turn at some bifurcation positions into completely different dispersion branches. These transitions correspond to mode couplings. For example, the faster sound wave (alpha-particle mode) couples with the Alfvén/ion-cyclotron wave of the protons at high wavenumbers, whereas the ion-acoustic wave couples with the drift-deformed Alfvén/ion-cyclotron wave of the alpha particles. The Rmode couples with the ion gyration at $\omega=1$ but, interestingly enough, not with the alpha-particle beam mode. It shows a break at $\omega=1$ and then continues above that frequency.

Two additional linear branches occur with merely constant phase speeds of $v \approx$ 


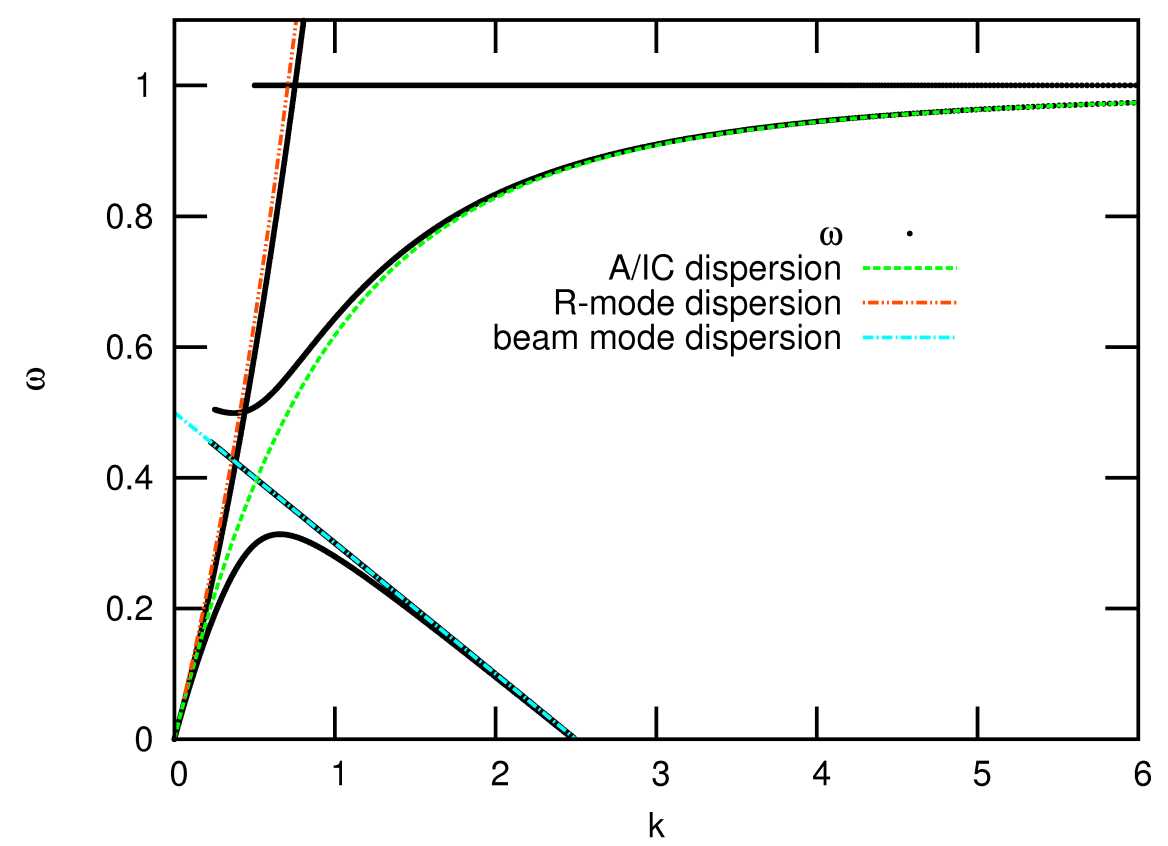

Figure 3. Dispersion relation for a plasma consisting of protons, electrons, and drifting alpha particles. The beam mode occurs for drifting alpha particles.

0.13 and $v \approx 0.03$. Such linear acoustic modes as these two were also found by Mann et al. (1997) for a warm plasma. The wave phase speed of these modes is usually determined by

$$
\begin{aligned}
& v_{\mathrm{Ph} 1}=\frac{\gamma_{\mathrm{p}} k_{\mathrm{B}} T_{\mathrm{p}}+\gamma_{\mathrm{e}} k_{\mathrm{B}} T_{\mathrm{e}}}{m_{\mathrm{p}}} \\
& v_{\mathrm{Ph} 2}=\frac{\gamma_{\alpha} k_{\mathrm{B}} T_{\alpha}}{m_{\alpha}}
\end{aligned}
$$

for a plasma with $n_{\alpha} \ll n_{\mathrm{p}}$. The first is the so-called ion-acoustic speed, the second is the sound speed of the alpha-particle component. In our case, these velocities are given by $v_{\mathrm{Ph} 1}=0.13$ and $v_{\mathrm{Ph} 2}=0.23$. The first one corresponds perfectly with the fast (steeper line) sound wave that we have found. The drift velocity $v_{\mathrm{d}}$ of the alpha particles is the reason for the deviation of the second linear mode. In the non-drifting reference case, the two velocities match $\left(v_{\mathrm{Ph} 2}-v_{\mathrm{d}}=0.03\right)$. If the numerical dispersion code is applied to a plasma without drift, the second sound wave branch is directly found at $v=v_{\mathrm{Ph} 2}$ (not shown here).

The two sound wave modes do not appear in the homogeneous plasma. Remember, that the phase speed $v$ is a free parameter in our calculation, and a wavenumber is given by the eigenvalues obtained for each $v$. If a wave is not dispersive, and therefore has a constant phase speed, it is not possible to calculate the full set of possible $k$-values that belong to this $v$. The sound waves exist already in the homogenous case; however, they become visible only after becoming dispersive due to the mode coupling. This type of wave becomes only dispersive for non-zero beta and in the 


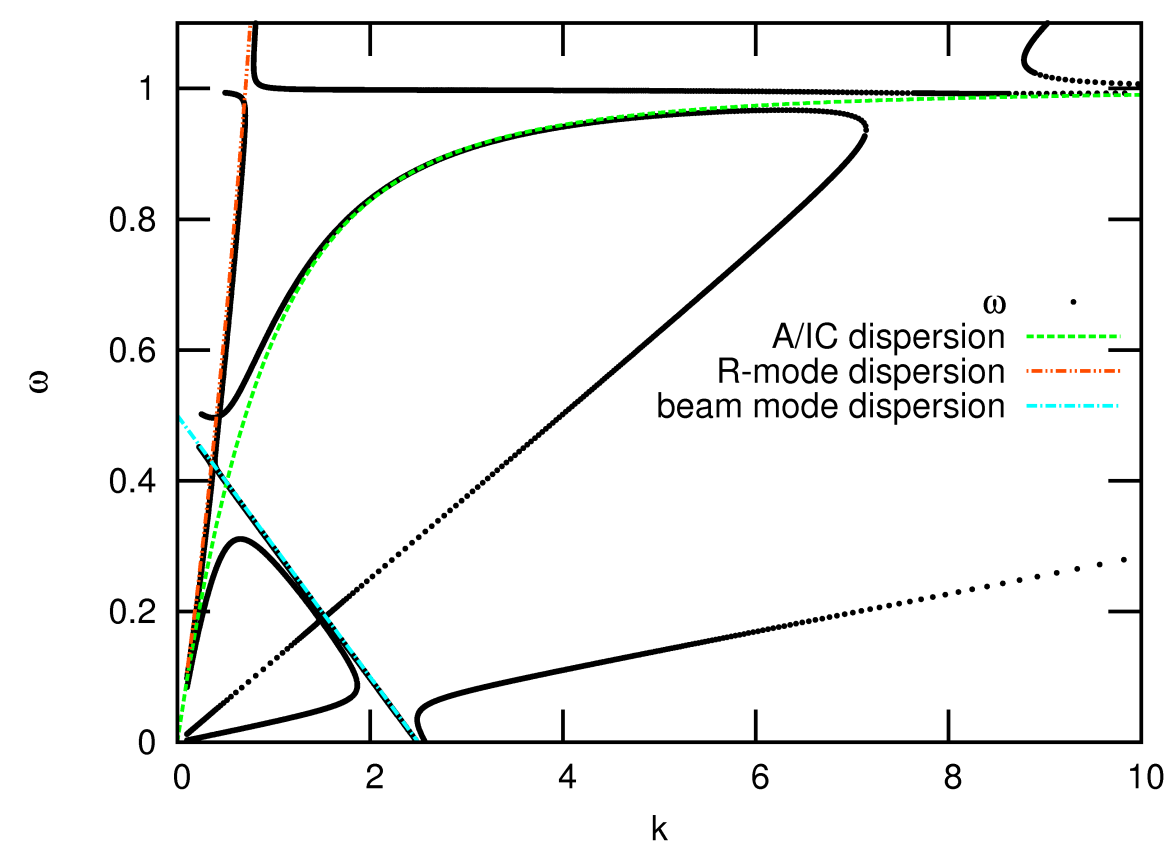

Figure 4. Dispersion relation for a plasma consisting of protons, electrons, and drifting alpha particles. The frequency $\omega$ is shown in dependence on the wave number $k$ in dimensionless units. The derived A/IC branch deviates from the cold dispersion branch at low and high wave numbers. Mode coupling between the various branches occurs as discussed in the text.

presence of the background wave. Also above the corresponding gyrofrequencies $\omega=1$ and $\omega=1 / 2$, the branches continue with their constant phase speed.

The waves on all branches have a compressive component, owing to the nonvanishing electric fields $e_{\mathrm{n}}$ and $e_{j \mathrm{n}}$. The real part of the eigenvalues $\lambda_{i}$ is always zero, i.e., none of the compressive modes is unstable in this case.

Note that it is the amplitude of the background wave which mainly determines the strength of the mode coupling and, therefore, the position and shape of the deformation of the ion-cyclotron branch. For higher amplitudes, the branch turns earlier away from the A/IC dispersion branch and, hence, the phase speed of the linear mode increases already at lower $k$-values. The plasma beta determines the phase speeds of the linear branches. Higher betas lead to higher phase speeds of these modes. The phase speeds can be adjusted relative to each other by choosing different betas for the individual species. However, the overall topology of the dispersion branches is not significantly changed by assigning different beta values to the different species.

However, if beta is chosen to be greater than one, the situation changes tremendously. The sound waves become unstable. In Fig. 5a, the dispersion is shown for a situation with $\beta_{j}=1.2$. The growth rate of the modes is shown in Fig. 5b.

Mode coupling between the sound wave and the whistler wave mode leads to an instability that occurs at $k \approx 0.8 \ldots 1.6$. Interestingly enough, this range is the ion gyroradius scale for moderate plasma betas close to one. This growing instability can therefore provide wave energy to the dissipative regime by means 

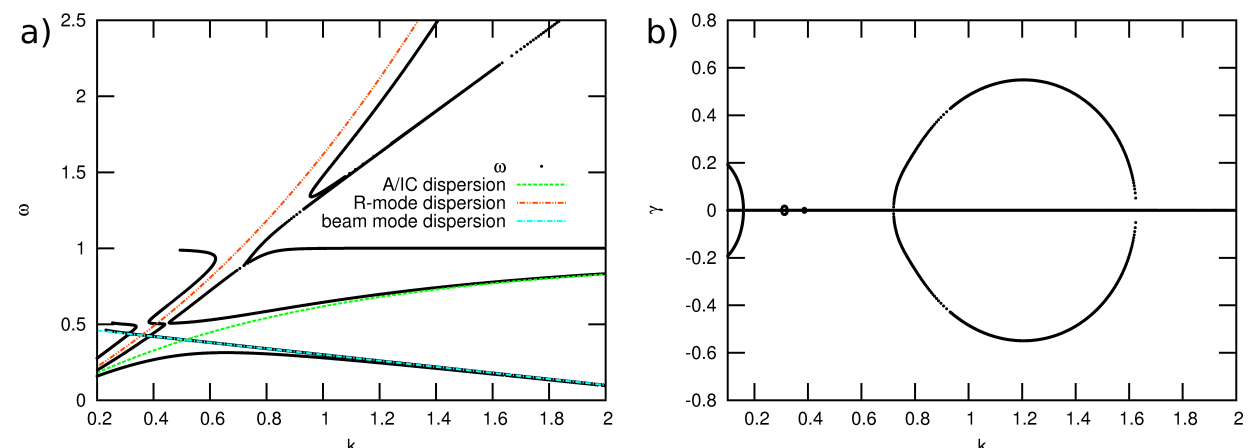

Figure 5. a) Dispersion relation for a plasma with $\beta=1.2$. b) Imaginary part of the frequency depending on $k$.

of Landau resonance, since the particle distribution has a significant number of particles at the corresponding wave speed of about $v \approx 1.36$ for a plasma with $\beta_{j} \gtrsim 1$. This instability vanishes if the background wave is absent, i.e., in the homogeneous case. For higher beta values, the position of the instability is shifted to higher wavenumbers.

\section{Conclusions}

As expected, the inhomogeneity of the background wave field leads to a deformation of the standard normal modes in the plasma. All of the discovered waves have a compressive component. Compressibility and inhomogeneity of the background plasma are the reasons for the new effects in the dispersion properties. The initially non-compressive Alfvén/ion-cyclotron waves become slightly compressive due to the inhomogeneous background, and are therefore able to interact with the protons via their electrostatic field components, in addition to the cyclotron resonance enabled by their transverse components. The quantitative details of these wave-particle interactions cannot be treated in the fluid description, since they are purely kinetic processes. Therefore they require a kinetic Vlasov treatment at least in the frame of quasi-linear theory and are, therefore, beyond the scope of this work. The kinetic refinement of the dispersion analysis of circularly polarised waves of the same type was described by Stenflo (1976), who also showed how relativistic effects and compressibility can modify the dispersion of large-amplitude waves in multi-fluid theory.

The non-constant (due to the presence of the pump wave) background leads to nonlinear mode couplings between some of the linear wave modes. It also leads to the excitation of initially non-dispersive modes, such as the ion-acoustic wave or the alpha-particle sound wave, yet now with $k$-dependent wave speeds.

The acoustic modes can grow at wavenumbers around the ion gyration scale under certain conditions and for adequate parameters. Thus, they are good candidates for a longitudinal electrostatic wave field with which particles can undergo Landau-resonant wave-particle interactions. Kinetic Alfvén waves (KAWs) have recently been discussed as another possible reason for this kind of interaction. KAWs owe their compressibility to the oblique geometry of their propagation with respect to a constant background field. In this study, we can show that also waves 
with purely parallel propagation can grow nonlinearly at the corresponding resonant wavenumbers, provided that the background has a non-trivial - but quite reasonable - magnetic field configuration. These two mechanisms are quite different in nature and should be further investigated and compared with each other.

The instability of ion-acoustic modes coupled to transverse modes were also discussed in studies of the parametric decay of large-amplitude pump waves and evaluated by numerical simulations (Araneda et al., 2007; Valentini and Veltri, 2009). In these studies, also instabilities of longitudinal waves at high wavenumbers are found, however at different background parameters. These waves are usually interpreted as results of nonlinear wave-wave interactions. Our linearised wave equations, however, can explain a similar growth of daughter waves at higher frequencies than the initial pump wave frequency as a consequence of compressibility and a non-uniform background. This mechanism can thus be understood as a new spectral transfer process of plasma fluctuations. It is very similar to the decay instability, which is found with the characteristic $k \gg k_{0}$.

Our eigenvector analysis still keeps the freedom to choose the amplitudes $\alpha_{i}$ in Eq. (2.19). This means that a wave can in general only occur if its amplitude is finite. It is beyond the scope of this work to investigate how the discovered wave branches can be excited in a real plasma. The linear dispersion analysis can only show possible normal modes. The discovered instability leads to growth in the initial phase only until nonlinear couplings and perhaps saturation occur. Yet unstable modes can grow from the thermal noise (that is constrained as a finite eigenvector of the system of equations) with a certain finite amplitude. In the presence of a large-amplitude wave, this thermal noise can lead to growth according to the calculated growth rate $\gamma$ at the gyroradius scale range for $\beta \gtrsim 1$. Maybe, full nonlinear calculations can investigate the further evolution and possible nonlinear excitations of such modes. The linear approximation appears to reflect the basic situation well (Lehe et al., 2009).

In the future, also other background conditions should be inquired. The above chosen background is one of the simplest inhomogeneous conditions. It is important to note that in our approach the background has to change slowly in dependence upon the position $\xi$. Otherwise, the use of a fixed phase speed $v$ is not possible anymore, and then the present approximation needs to be changed. The original set of coupled wave equations remains valid, yet another mathematical treatment is required to cope with their nonlinearity.

\section{Acknowledgement}

D. V. is grateful for financial support by the International Max Planck Research School (IMPRS) on Physical Processes in the Solar System and Beyond.

\section{References}

Akhiezer, A. I., Akhiezer, I. A., Polovin, R. V., Sitenko, A. G. and Stepanov, K. N. 1975 Plasma electrodynamics - Vol.1: Linear theory. Oxford, UK: Pergamon Press.

Antonucci, E., Dodero, M. A. and Giordano, S. 2000 Solar Phys. 197, 115.

Araneda, J. A., Marsch, E. and Viñas, A. F. 2007 J. Geophys. Res. 112, 4104.

Bale, S. D., Kellogg, P. J., Mozer, F. S., Horbury, T. S. and Reme, H. 2005 Phys. Rev. Lett. 94, 215002. 
Belcher, J. W. and Davis, L., Jr. 1971 J. Geophys. Res. 76, 3534.

Brodin, G., Shukla, P. K. and Stenflo, L. 2008 J. Plasma Phys. 74, 99.

Bruno, R. and Carbone, V. 2005 Living Rev. Sol. Phys. 2, 4.

Chandran, B. D. G. 2005 Phys. Rev. Lett. 95, 265004.

Chandran, B. D. G., Li, B., Rogers, B. N., Quataert, E. and Germaschewski, K. 2010 Astrophys. J. 720, 503.

Chandran, B. D. G., Quataert, E., Howes, G. G., Xia, Q. and Pongkitiwanichakul, P. 2009 Astrophys. J. 707, 1668.

Coleman, P. J., Jr. 1968 Astrophys. J. 153, 371.

De Pontieu, B., McIntosh, S. W., Carlsson, M., Hansteen, V. H., Tarbell, T. D., Schrijver, C. J., Title, A. M., Shine, R. A., Tsuneta, S., Katsukawa, Y., Ichimoto, K., Suematsu, Y., Shimizu, T. and Nagata, S. 2007 Science 318, 1574.

Galeev, A. A. and Oraevskii, V. N. 1963 Sov. Phys. Doklady 7, 988.

Gary, S. P., Smith, C. W. and Skoug, R. M. 2005 J. Geophys. Res. 110, 7108.

Goldstein, M. L. 1978 Astrophys. J. 219, 700.

Heuer, M. and Marsch, E. 2007 J. Geophys. Res. 112, 3102.

Hollweg, J. V. and Isenberg, P. A. 2002 J. Geophys. Res. 107, 1147.

Jian, L. K., Russell, C. T., Luhmann, J. G., Strangeway, R. J., Leisner, J. S. and Galvin, A. B. 2009 Astrophys. J. 701, L105.

Kasper, J. C., Lazarus, A. J. and Gary, S. P. 2008 Phys. Rev. Lett. 101, 261103.

Kellogg, P. J., Bale, S. D., Mozer, F. S., Horbury, T. S. and Reme, H. 2006 Astrophys. J. 645, 704.

Kohl, J. L., Noci, G., Cranmer, S. R. and Raymond, J. C. 2006 Astron. Astrophys. Rev. 13, 31.

Lashmore-Davies, C. N. and Stenflo, L. 1979 Plasma Phys. 21, 735.

Lehe, R., Parrish, I. J. and Quataert, E. 2009 Astrophys. J. 707, 404.

Mann, G., Hackenberg, P. and Marsch, E. 1997 J. Plasma Phys. 58, 205.

Marsch, E. 2006 Living Rev. Sol. Phys. 3, 1.

Marsch, E. and Tu, C. 2001 J. Geophys. Res. 106, 8357.

Marsch, E. and Verscharen, D. 2011 J. Plasma Phys. 77, 385.

Murtaza, G. and Shukla, P. K. 1984 J. Plasma Phys. 31, 423.

Podesta, J. J., Borovsky, J. E. and Gary, S. P. 2010 Astrophys. J. 712, 685.

Press, W. H., Teukolsky, S. A., Vetterling, W. T. and Flannery, B. P. 1992 Numerical recipes in FORTRAN. The art of scientific computing. Cambridge, UK: Cambridge University Press.

Schwenn, R. and Marsch, E. 1991 Physics of the Inner Heliosphere II. Particles, Waves and Turbulence. Berlin, Germany: Springer-Verlag.

Sharma, R. P. and Shukla, P. K. 1983 Phys. Fluids 26, 87.

Sonnerup, B. U. Ö. and Su, S.-Y. 1967 Phys. Fluids 10, 462.

Stenflo, L. 1976 Physica Scripta 14, 320.

Stenflo, L. and Shukla, P. K. 2007 In: Handbook of the Solar-Terrestrial Environment (ed. Kamide, Y. \& Chian, A. C.-L.) p. 311.

Stix, T. H. 1992 Waves in plasmas. New York, USA: American Institute of Physics.

Tu, C. and Marsch, E. 1994 J. Geophys. Res. 99, 21481.

Tu, C. and Marsch, E. 1995 Space Sci. Rev. 73, 1.

Valentini, F. and Veltri, P. 2009 Phys. Rev. Lett. 102, 225001.

Verdini, A., Velli, M., Matthaeus, W. H., Oughton, S. and Dmitruk, P. 2010 Astrophys. J. Lett. 708, L116. 